\title{
Building Theoretical Foundations for Electronic Governance Benchmarking
}

\author{
Adegboyega Ojo, Tomasz Janowski, and Elsa Estevez \\ United Nations University - International Institute for Software Technology \\ Center for Electronic Governance \\ P.O. Box 3058, Macao SAR \\ $\{a o, t j, e l s a\}$ @iist.unu.edu
}

\begin{abstract}
The success of the electronic governance (EGOV) benchmarking has been limited so far. Lacking a theory to integrate existing conceptualizations has made the acquisition and sharing of knowledge produced by different benchmarking exercises difficult. In order to address this problem, this paper: 1) explains the nature of the EGOV benchmarking activity though a wellestablished theoretical framework - Activity Theory, 2) applies the framework to carry out a mapping between a number of existing EGOV benchmarking conceptualizations, 3) develops an unified conceptualization based on these mappings and 4) validates the resulting model though a real-life national EGOV strategy development project. The use of the Activity Theory in the paper has enabled defining and relating initial dimensions of the EGOV benchmarking activity, and mapping the dimensions present in existing conceptualizations. This not only created a unifying theoretical basis for conceptualizing the EGOV benchmarking activity but allowed learning from and integrating existing conceptualizations. The work impacts on the EGOV benchmarking practice by enabling a logical design of the activity, and contextually correct understanding of existing EGOV benchmarking results with respect to their intended usage.
\end{abstract}

Keywords: Electronic Governance, Benchmarking, Activity Theory.

\section{Introduction}

Since it was introduced by Xerox Corporation over two decades ago [2][10], benchmarking has become a well established tool for improving organizational performance and competitiveness particularly in the private sector. With increasing focus on performance management and continuous improvement in government, benchmarking has been also accepted as a useful management instrument in the public sector [16]. In particular, the international benchmarking series like the United Nations e-Government Survey, Accenture e-Government survey or the European Union e-Government Study are well known in the EGOV domain [7][8][22].

The concept of benchmarking has received various definitions. For instance, [17] refers to the process of evaluating and applying best practices in order to improve performance while [15], in the EGOV context, defines benchmarking as a systematic comparison of the performance of (parts of) organizations and their similar services, 
processes and routines, based on predetermined indicators, with the goal of improving performance by learning from one another. As a concept, benchmarking has evolved significantly over the years. Today, competitive, process, strategic and network benchmarking are all carried out in private and public sectors, and at the international, regional and national levels [17][18][24], with contemporary practice shifting from model learning (i.e. learning what) towards process learning (i.e. learning how) and adaptive learning (i.e. learning to change) [17]. For any organization involved in the benchmarking exercise, the final pragmatic goal is identifying learning points and understanding how what has been learnt could make the organization better [2].

As a research domain, benchmarking research is fairly mature. For instance in 2002 there were over 350 publications on benchmarking, shared between foundations (46\%) and applications (43\%) [9]. In February 2011, the authors' search in the Scopus database (www.scopus.com) of the articles with "benchmarking" in the title resulted in over 4000 publications and a dedicated journal: Benchmarking - An International Journal. Despite this, [3] notes the scarcity of literature devoted to the conceptual and practical problems of EGOV benchmarking. Indeed, the Scopus database search produced only 40 publications in this area, congruent with the finding by Yasin [29] that only $1.8 \%$ of the benchmarking literature is associated with the public sector.

The need for a better theoretical and conceptual foundation for benchmarking in general and for EGOV benchmarking in particular were pointed out in [10][17][20]. Specifically, [29] asserted in 2002 that the academic community was lagging in developing models and frameworks that integrate many aspects of organizational benchmarking. Since then, efforts aimed at providing the required conceptual foundations have been documented in [3][8][12][15], albeit using different terminologies and levels of abstraction. The resulting conceptual multiplicity makes the integration of research findings associated with different conceptualizations difficult, and leaves the need for more theory development unresolved [19].

Many of the reported issues relating to the EGOV benchmarking practice are arguably caused by a mismatch between the original purpose and subsequent use of the benchmarking results [25]. For instance, it is common for EGOV benchmarking series to rank countries in very different ways. In order for governments to learn from and exploit these differences, they must understand how the goals, scope, measures, etc. used by different series are related, how to correctly and meaningfully interpret and reason about different benchmark data with respect to specific exploitation goals, e.g. to inform EGOV strategy formation for better EGOV global positioning.

To address the problem above, this paper applied Activity Theory [1] as a unifying framework. A tool for better understanding human activities taking place within social and organizational contexts, Activity Theory has been widely used in learning, organizational analysis, design of interactive systems, enterprise engineering and others [1][23][27]. As EGOV benchmarking is an activity situated within the government context, carried out by policy makers, strategists and researchers to achieve certain learning-oriented outcomes, it is intuitively amenable to Activity Theory-based analysis. Indeed, [23] shows how Activity Theory can be applied as a mapping and integrative framework for enterprise ontologies. Here, Activity Theory is used to align existing conceptualizations of EGOV benchmarking, and to integrate them into a unified conceptualization which, well-grounded in theory, enables specification and analysis of EGOV benchmarking. The paper also shows how this 
unifying conceptualization was build and applied in analyzing a concrete benchmarking activity within a real-life national EGOV strategy project.

Our contributions to EGOV benchmarking research and practice are as follows: 1) providing a theory-based unifying model which identifies and relates the core dimensions of the "EGOV benchmarking" concept, and 2) enabling detailed design of the EGOV benchmarking activity as well as detailed profiling of existing EGOV benchmarking initiatives to guide the use of their associated results.

The rest of the paper is organized as follows. Section 2 provides an overview and analysis of existing EGOV benchmarking conceptualizations. Section 3 presents the methodology adopted to guide this research, followed by the presentation of Action Theory as adopted theoretical framework in Section 4. The Activity Theory-based benchmarking model and the process of mapping and integrating existing conceptualizations using this model, resulting in the unified EGOV benchmarking conceptualization, is described in Section 5. Section 6 validates this conceptualization though a real-life national EGOV strategy project, Section 7 discusses the findings of the paper, and Section 8 provides some conclusions.

\section{Related Work}

This section provides an overview and discussion of eight EGOV benchmarking conceptualizations. Section 2.1 presents five research-oriented conceptualizations, followed by three practice-oriented conceptualizations in Section 2.2, and some observations about these conceptualizations in Section 2.3.

\subsection{Research-Oriented Conceptualizations}

Among the research-oriented conceptualization presented below, [17][25] were published in measurement-related journals, [3] in a public administration journal and [8][15] in EGOV journals. Among them [3][8][15][25] address EGOV benchmarking, while [17] addresses general benchmarking.

Kyro et al. [17] argue for the need to update the traditional concept and forms of benchmarking to address contemporary issues. To this end, the activity is described using three dimensions: benchmarker - who is benchmarking; target - what is to be benchmarked; and partner - with whom the subject will be benchmarked.

Banister [3], to answer the questions of usefulness and beneficiaries of EGOV benchmarking, discusses conceptual issues in EGOV benchmarking and proposes three questions for any benchmarking exercise: what is the purpose, what is to be measured, and what type of benchmarking should be carried out.

Saleem [25] provides a conceptual framework to guide governments in assessing the applicability of EGOV benchmarking as a driver for EGOV initiatives. The framework employs four dimensions to analyze international benchmarking reports: context, methodology, benchmarking type, and sociological paradigm.

Codagnone et al. [8], drawing from concrete practice, describe a conceptual framework for EGOV benchmarking based on the European Commission's benchmarking projects. The framework provides three dimensions in the form of questions: what to measure, how to measure, and for whom to measure. 
Lastly, Jansen et al. [15] describes a model-based method aimed at improving the practice of EGOV service benchmarking, with fives benchmarking dimensions: goal, respondents, indicators, methods and infrastructure; and three levels of analysis: benchmark partner level, organizational level, and specific service level.

\subsection{Practice-Oriented Conceptualizations}

Among the practice-oriented conceptualizations [12][14][26] presented in this section, [14][26] focus respectively on benchmarking Information Society and EGOV within the European Union, while [12] provides concrete EGOV benchmarking guidelines. The three conceptualizations are described as follows.

IANIS [14] identifies a number of dimensions for carrying out benchmarking in support of EU regional strategies for developing the information society: what to collect data about, when to collect data, about whom to collect data, from whom to collect, how to collect secondary data, how to collect fresh data, at what level of aggregation to compare regions, and how to process benchmark data.

EUeGovBe [26] is a related but specialized framework for EU e-Government Benchmarking. The framework presents three detailed dimensions: guiding principles and policy, benchmarking method and reporting and learning.

Heeks [12] provides detailed guidelines on how to answer the questions of purpose, subject, method and presentation of EGOV benchmarking, together with conceptual models to support the operationalization of the benchmarking exercise.

\subsection{Observations}

As we can see in Sections 2.1 and 2.2, the purpose of conceptualizations varies, from analysis of benchmarking initiatives, through effective use of benchmarking results, to standardization of the benchmarking practice and tracking of policy implementations.

Unlike traditional benchmarking which is carried out by an organization to learn from others, and thus improve its own operations, EGOV-related benchmarking is carried out mostly by third-party organizations [3]. Among the reviewed conceptualizations, only [15][17] position government in the role of a benchmarking entity. This may affect the expectations of the learning outcomes in the EGOV benchmarking. For instance, only [12][26] explicitly address the learning objectives.

Considering our goal, none of the research-oriented EGOV conceptualizations, except [17], has been formally derived or associated with specific theory or existing conceptualization. The conceptualizations are also relatively incomplete, compared to generic ones [17], lack validity information, and mappings between them do not exist.

\section{Methodology}

By conceptualization we mean the process of transforming a theoretical construct, for instance "EGOV benchmarking", into observable or measured concepts, for instance the "benchmark method" with "scope", "data gathering method"," type of analysis" and "research paradigm" elements [19]. By theoretical construct we mean a set of conceptual terms used to define a phenomenon of theoretical interest [11], used as fundamental elements to drive research and practice in a given field [15]. 
Conceptualization is essentially carried out in two ways: theoretical - starting from a theoretical construct and then operationalizing and measuring it, or observational starting with observations and relating them through models. Adopting the former approach, this work starts with the EGOV benchmarking construct but, to minimize possible inconsistencies caused by different operationalizations, constrains the resulting conceptual model in a way similar to the hybrid approach described in [19].

The methodology prescribes two basic steps grounded in Activity Theory [19] and depicted in Table 1: inductive - determine the dimensions of the construct, and deductive - relate the dimensions into a conceptual model. The former starts by considering benchmarking simply as an activity undertaken by human agents for a certain purpose. This enables the grounding of benchmarking in Activity Theory [23][27] and identification of initial dimensions of the benchmarking construct, used to discover domain-specific dimensions from the conceptualizations in Section 2. The task is analogous to ontology mapping [5] or integration of constructs [17]. The deductive step systematizes the dimensions, creating a conceptual model for EGOV benchmarking, where the meaning of dimensions and the relationships between them are consistent with those of the underlying Activity Theory-based benchmarking model. The approach is similar to [17] - mapping and analyzing benchmarking conceptualizations to build a more detailed conceptualization, and [23] - using Activity Theory to synthesize dimensions of an enterprise ontology.

Table 1. EGOV Conceptualization Methodology

\begin{tabular}{lll}
\hline & Inductive Step & Deductive Step \\
\hline Activity Theory & $\begin{array}{l}\text { Generic dimensions for the benchmarking } \\
\text { construct based on Activity Theory }\end{array}$ & $\begin{array}{l}\text { Activity Theory-based } \\
\text { model for benchmarking }\end{array}$ \\
$\begin{array}{ll}\text { EGOV-related } \\
\text { Conceptualizations }\end{array}$ & $\begin{array}{l}\text { Domain-specific EGOV benchmarking } \\
\text { dimensions with existing conceptualizations }\end{array}$ & $\begin{array}{l}\text { EGOV with domain- } \\
\text { specific dimensions }\end{array}$ \\
\hline
\end{tabular}

\section{Theoretical Framework - Activity Theory}

The Activity Theory is a tool for better understanding human activities within social and organizational contexts, attempting to link human actions with the relevant contextual elements [28]. The theory enables assessing the factors that influence the performance of an Activity by a Subject, using Artifacts, subject to certain Rules, and divided among members of a Community to act in specific Roles, to accomplish an Object and ultimately an Outcome that influences an organization [23]. Figure 1 depicts the model of an activity derived from Engestrom [30] and the original in [28].

As a unit of analysis, activities are [28]: 1) mediated using artifacts and tools; 2) pragmatic as they are driven by objects and motives; 3) situated in specific time, space and community; 4) provisional as they evolve continuously; and 5) contested since they involve varied interests, viewpoints and perspectives and often give rise to contradictions both within and between activities (dialectical perspective). 


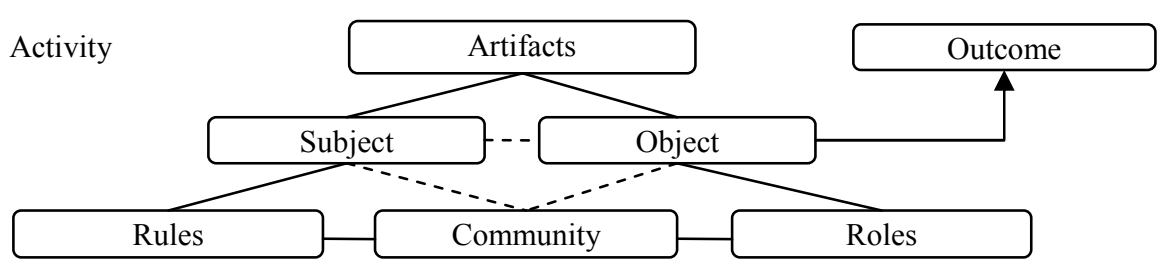

Fig. 1. Activity Representation in Activity Theory

The theory has been used in various domains including learning, organizational analysis, design of interactive systems and enterprise engineering [1][23][28].

\section{Activity Theory-Based EGOV Benchmarking}

This section presents the details of the EGOV benchmarking conceptualization based on the Activity Theory. Section 5.1 shows how the theory helps synthesize the generic dimensions of the benchmarking construct. Section 5.2 interprets the eight EGOV benchmarking conceptualizations in Section 2 against the generic dimensions and helps discover new dimensions. Section 5.3 organizes all dimensions into an Activity Theory-based EGOV benchmarking model, followed by the operationalization of the model through a case study in Section 5.4 and its validation in Section 5.5.

\subsection{Activity Theory-Based Generic Benchmarking Model}

The relevance of the Activity Theory to EGOV benchmarking rests upon two arguments. First, benchmarking is an activity that should be carried out in a context $[2][4][6][16][15]$. Second, as the context is often inscribed into EGOV system design, such inscriptions can mismatch the actual deployment context creating a contextual collision that could lead to EGOV failure [13]. To operationalize this connection, consider that the benchmarking activity (Activity) is carried out: by a benchmarker (Subject); using a certain benchmarking approach (Artifacts); subject to certain

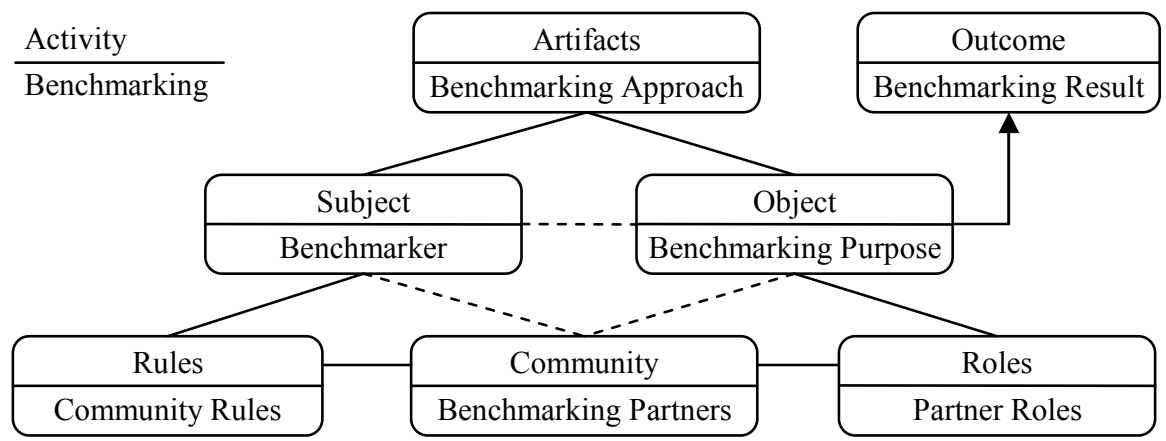

Fig. 2. Activity Theory-Based Benchmarking Model 
benchmarking rules (Rules); and involving benchmarking partners (Community) with their commitments and roles (Roles); to achieve a certain benchmarking purpose (Object) and eventually the expected benchmarking results (Outcome). The resulting Activity Theory-based model for benchmarking is shown in Figure 2.

The model maps the eight generic concepts of the Activity Theory - Activity, Subject, Artifact, Object, Outcome, Community, Roles and Rules into the corresponding concepts in the benchmarking domain, as follows [1][21][23]:

1. Activity - A form of "doing", it is the main object of concern in Activity Theory and usually comprises several actions. In the benchmarking domain, Activity is mapped into Benchmarking. It is specified as a collection of the specifications of the other seven elements defined below. Example is EGOV Benchmarking.

2. Subject - An individual or organization that undertakes an Activity. In the benchmarking domain, Subject is mapped into Benchmarker e.g. government organization, international EGOV ranking organization, EGOV researcher, etc.

3. Object - It is explored or transformed by Subject to motivate and achieve the goals of Activity. In the benchmarking domain, Object is mapped into Benchmarking Purpose, e.g. "to determine the source of good practice for citizenfocused mobile services" and determines the Benchmarks or measures.

4. Artifact - The material or conceptual tools that mediate actions of the Subject on the Object, produced by other activities. In the benchmarking domain, Artifact is mapped into Benchmarking Approach - processes, techniques and tools to support the benchmarking activity, e.g. online survey instruments.

5. Outcome - The final result of Activity obtained when pursuing Object, possibly serving as Artifact for another Activity. In the benchmarking domain, Outcome is mapped into Benchmarking Result, e.g. EGOV ranking or benchmarking report prepared by a government agency for a supervisory office.

6. Community - All partners (people or organizations) directly involved in Activity, sharing the Object with the Subject, and establishing a link between the Subject and Activity context. In the benchmarking domain, Community is mapped into Benchmarking Partners for data gathering, resourcing and joint implementation.

7. Rules - The norms - guidelines, code of conduct, heuristics and conventions that mediate cooperation within and participation of the Subject in the Community. In the benchmarking domain, Rules are mapped into Community Rules, e.g. open publication of data on public service delivery by Community partners.

8. Roles - Refer to how cooperation and specialization occurs in an Activity to achieve the Object. In the benchmarking domain, Roles are mapped into Partner Roles, e.g. commitment by Community partners to implement good practices.

This mapping identifies eight generic dimensions for the benchmarking construct: 1) Benchmarking, 2) Benchmarker, 3) Benchmarking Purpose, 4) Benchmarking Approach, 5) Benchmarking Result, 6) Benchmarking Partners, 7) Community Rules and 8) Partner Roles, which concludes the first task in our methodology.

\subsection{Activity Theory-Based Interpretation of EGOV Benchmarking}

This section interprets the eight EGOV benchmarking conceptualizations in Section 2 against the generic dimensions of the EGOV benchmarking construct in Section 5.1. 
This has two goals: 1) provide EGOV-specific semantics to the generic benchmarking dimensions and 2) discover new dimensions for the EGOV benchmarking construct to explain existing conceptualizations, beyond the explanatory power of the Activity Theory. The interpretation is carried out by explaining each dimension using defining terms of existing EGOV conceptualizations as well as seeking the terms that cannot be explained by the generic dimensions. The exception to this is the Benchmarking dimension, explained in terms of the remaining, but having no source in existing conceptualizations (except the current paper). The result is shown in Table 3.

The definitions of the various terms in Table 3 are as defined in their source conceptualizations. However, the informal nature of the conceptualizations was the cause of frequent ambiguity of terms, making the task of mapping terms across conceptualizations challenging. For instance, the term Scope is used in [12] to represent a range of benchmark measures, part of the Benchmark dimension, but also in [17] to represent Geographical Scope. Term disambiguation and re-naming was carried out by examining example uses of each term in the conceptualizations.

Interestingly, the Activity Theory-based generic benchmarking dimensions suffice for explaining all eight EGOV benchmarking conceptualizations. On the one hand, all dimensions can be explained using defining terms of existing EGOV benchmarking

Table 2. Interpreting EGOV Benchmarking Conceptualizations against Generic Dimensions

\begin{tabular}{|c|c|c|c|}
\hline No & Dimensions & Defining Terms & Conceptualizations \\
\hline 1 & Benchmarking & $\begin{array}{l}\text { Benchmarker; Benchmarking: Purpose, } \\
\text { Approach, Result, Partners; Community } \\
\text { Rules; Partner Roles }\end{array}$ & \\
\hline 2 & Benchmarker & $\begin{array}{l}\text { Nature of Organization, Organizational } \\
\text { Structure Type, Geographical Scope }\end{array}$ & $\begin{array}{l}\text { Kyro et al., Banister, } \\
\text { Saleem }\end{array}$ \\
\hline 3 & $\begin{array}{l}\text { Benchmarking } \\
\text { Purpose }\end{array}$ & Purpose, Policy, Priorities & $\begin{array}{l}\text { Heeks, Banister, Saleem, } \\
\text { Jansen et al., EUeGOVBe }\end{array}$ \\
\hline 4 & $\begin{array}{l}\text { Benchmarking } \\
\text { Approach }\end{array}$ & $\begin{array}{l}\text { Scope, Data Source/Method, Analysis } \\
\text { Type/Unit, Research Paradigm, Limitation, } \\
\text { Timing, Resources, Indicators, Underlying } \\
\text { Framework, Indicators, Execution }\end{array}$ & $\begin{array}{l}\text { Heeks, Banister, Saleem, } \\
\text { Codagnone et al., Jansen et } \\
\text { al., IANIS, EUeGOVBe }\end{array}$ \\
\hline 5 & $\begin{array}{l}\text { Benchmarking } \\
\text { Result }\end{array}$ & $\begin{array}{l}\text { Audience, Reporting, Access, } \\
\text { Dissemination }\end{array}$ & $\begin{array}{l}\text { Heeks, Codagnone et al., } \\
\text { EUeGOVBe }\end{array}$ \\
\hline 6 & $\begin{array}{l}\text { Benchmarking } \\
\text { Partners }\end{array}$ & $\begin{array}{l}\text { Nature of Organization, Organizational } \\
\text { Structure Type, Geographical Scope }\end{array}$ & $\begin{array}{l}\text { Kyro et al., Jansen et al., } \\
\text { IANIS }\end{array}$ \\
\hline 7 & $\begin{array}{l}\text { Community } \\
\text { Rules }\end{array}$ & $\begin{array}{l}\text { Data Format Standards, Quality Control, } \\
\text { Data Privacy }\end{array}$ & IANIS \\
\hline 8 & Partner Roles & $\begin{array}{l}\text { Governance, Data Provider, Execution } \\
\text { Party }\end{array}$ & IANIS, EUeGOVBe \\
\hline 9 & Benchmark & $\begin{array}{l}\text { Benchmark Type, Stakeholder Scope, } \\
\text { Level of Government, Measure }\end{array}$ & $\begin{array}{l}\text { Kyro et al., Heeks, Saleem, } \\
\text { Codagnone et al., Jansen et } \\
\text { al., IANIS, Banister }\end{array}$ \\
\hline
\end{tabular}


conceptualizations, albeit to different extent. For example, the explanation of the Purpose, Community, Rules and Roles dimensions by existing research-oriented conceptualizations is weak. In fact, only [14] explicitly addresses the Rules dimension in its conceptualization. On the other, all defining terms of existing EGOV benchmarking conceptualizations could fit within one of eight generic dimensions except one - Benchmark. Benchmark is thus added as a new discovered dimension.

\subsection{Activity Theory-Based EGOV Benchmarking Model}

Figure 3, elaborating on the model in Figure 2, provides a more precise meaning for different dimensions of the EGOV benchmarking construct. The model adds centrally the Benchmark dimension, and dependencies between dimensions. The grounding in Activity Theory enables the exploitation of the theory in the EGOV benchmarking context. For instance, the Subject element in Activity Theory brings into focus the Benchmarker concept missing in many EGOV benchmarking conceptualizations.

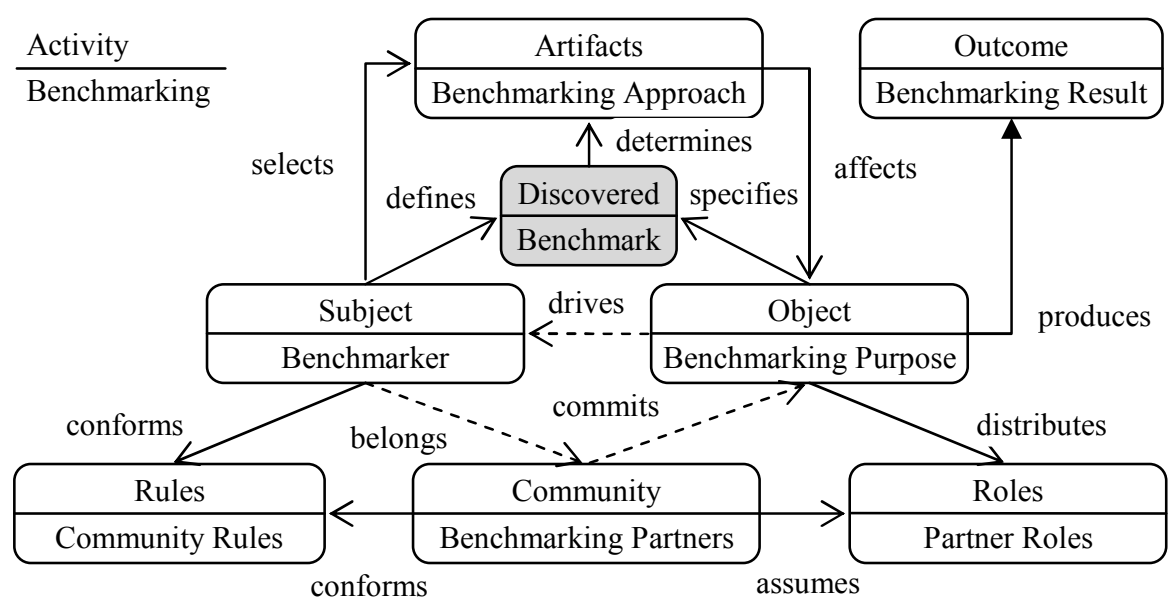

Fig. 3. Activity Theory-Based EGOV Benchmarking Model

\subsection{Operationalizing Activity Theory-Based EGOV Benchmarking Model}

This section describes how the EGOV benchmarking model defined in Section 5.3 was applied to guide a strategic benchmarking exercise within a national EGOV strategy development project implemented by the authors and a government partner. The aim of the exercise was to provide strategies and transferrable good practices in the area of EGOV (and e-participation) infrastructure. We highlight below the analyses made possible by the Activity Theory-based EGOV benchmarking dimensions, not provided or sufficiently explicated in existing conceptualizations.

Outcome - The project focused on two possible outcomes of the benchmarking exercise: producing strategies to lead to fundamental improvements in the country's EGOV program, and raising the country's international EGOV ranking to stimulate more funding. This prompted to carefully specify what information is sought. 
Object - Considering the expected outcomes, the project elaborated and prioritized concrete objectives of the benchmarking exercise. In reaching the final decision of focusing on fundamental EGOV improvements and with increased country ranking as a secondary outcome, the implicit dialectical nature of Activity Theory was exploited.

Community - The notion of dynamically-changing Community with current state of Activity was applied in determining benchmarking partners through stakeholder analysis. The project developed community profiles to identify, adopt and utilize good practices e.g. a country with similar socio-economic and development condition serving as key sources of good practices in EGOV infrastructure development.

Roles - Based on the results of stakeholder analysis, each member of the identified Community was assigned a concrete Role for instance: data provider, benchmarking partner, best practice transfer facilitator, external user, etc.

Activity - Activity was treated as a context for developing various profiles for the benchmarking exercise, consisting of short statements and placeholders (meta-data) on different dimensions identified in the EGOV benchmarking model.

In a summary, the operationalization experience produced two main insights. First, the Activity, Object and Outcome dimensions enabled detailed profiling of the benchmarking exercise, addressing traditional concerns related to poor specification of benchmarking results [3] and insufficient information to assist users in making effective use of them [14]. Second, the model provided a theoretical framework to enable reasoning about the design of the benchmarking exercise, e.g. the composition of Community vis-à-vis the expected Outcome and cost, or policy and political interests vis-à-vis the impact on long-term organization and institutional goals.

\subsection{Validating Activity Theory-Based EGOV Benchmarking Model}

Two basic validation obligations identified for this work are: 1) the soundness of use of Activity Theory as a basis for conceptualizing the benchmarking activities, and 2) the validity of our conceptualization in terms of the process and resulting dimensions. Concerning the first obligation, we argue in Section 5.1 that benchmarking is fundamentally a context-based activity and shares inherent features of the activities defined in the Activity Theory. Concerning the process part of the second obligation, we explained in Section 3 how this work follows a well-established approach to conceptualization [21][19]. We could also adopt the formal conceptualization practice through ontologies. In fact, our mapping and integration process is analogous to a typical ontology mapping and integration exercises, and similar to [25] which also relies on Activity Theory as its base theory. Concerning the dimension part of the second obligation, the mapping of Activity Theory-based dimensions to equivalent dimensions across existing conceptualizations provides a form of validation or cross validation in terms of the relevance of the dimensions. Empirical validation e.g. vertical validity [11][29] of the dimensions and the established relations are planned as part of our future work. However, the use of our conceptualization in a concrete project in Section 5.4 is a first step in establishing its validity empirically. 


\section{Discussion}

So far, the proposals for improving the EGOV benchmarking practice focused mainly on finding better measures and associated indicators. Lately, the emphasis has been on situating the EGOV benchmarking in well-defined policy [6][28] or organizational [16] contexts, and benchmarking the EGOV backend [17][27]. Our first proposition (P1) is therefore that a conscious contextual embedding is critical to EGOV benchmarking in view of its purpose and to enable its exploitation.

The next challenge is how to bridge different contexts to enable accumulation of knowledge from cases, facing lack of a unifying theory to guide progress in the field [31] and methodological pluralism characteristic of e-Government measurement [8]. Thus our second proposition (P2) is that the availability of a unifying framework for EGOV benchmarking is essential to advancing its theory and practice.

In line with the second proposition, the paper shows that Activity Theory can play the role of a top-level integrative conceptualization for EGOV benchmarking, similar to the development of Activity Theory-based Enterprise Ontology in [25]. This leads to our third proposition (P3) that Activity Theory provides a useful framework for understanding and improving the EGOV benchmarking practice particularly through its focus on the context and purpose of the benchmarking activity. This is particularly important in view of the challenges facing public sector and EGOV benchmarking: determining time and cost [10][16][31], assessing the impact of benchmarking on an organizations [10] and ensuring that the benchmarking results are used correctly [27].

Further evidence in support of P3 is the possibility to address the correct use of benchmarking results through so-called using Activity Theory-based inter-activity systems [12] or boundary objects connecting multi-activity subjects [30]. The former also appears applicable to the regional benchmarking frameworks where either the outcomes or artifacts are shared among ongoing benchmarking activities.

Given its inherent limitations [30] and as Activity Theory evolves to address emerging organizational challenges, the range of its application in the benchmarking domain will have to be discovered over time.

\section{Conclusion}

The current EGOV benchmarking practice suffers from the lack of a theoretical framework to facilitate the accumulation of knowledge in the domain when carrying out different benchmarking exercises. This paper makes a step towards building a theoretical framework for EGOV benchmarking which relies on Activity Theory and which unifies existing conceptualizations. This effort opens up the possibility of exploring relationships between the dimensions towards domain theory building. For benchmarking practice, the model provides a comprehensive high-level framework for designing and analyzing EGOV benchmarking activities. Practical application of the model has also shown that it offers analysis and insight which are not possible with existing conceptualizations, e.g. the notion of communities and conflicts between the different interests of their members. Our future work includes applying the developed conceptual model to analyze and profile more EGOV benchmarking cases, towards empirical validation, as well as exploiting recent developments and extensions in the Activity Theory to address specific benchmarking challenges. 


\section{References}

1. Almeida, A., Roque, L.: Simpler, Better, Faster, Cheaper, Contextual: Requirements Analysis for a Methodological Approach to Interaction Systems Development. In: Proceedings of the 8th European Conference on Information Systems (2000)

2. Ammons, D.N.: A Proper Mentality for Benchmarking. Public Administration Review 59(2), 105 (1999)

3. Bannister, F.: The Curse of the Benchmark: An Assessment of the Validity and Value of e-Government Comparisons. International Review of Administrative Sciences 73(2), 171188 (2007)

4. Bruder, A.J., Gray, E.M.: Public-Sector Benchmarking: A Practical Approach. Public Management 76(9), 9+ (1994)

5. Bruijin, J. de, Martin-Recuerda, F., Manov, D., Ehrig, M.: Ontology Merging and Aligning V1 (2003)

6. Capgemini, et al.: Method Paper 2010 Preparing the 9th Benchmark Measurement (June 2010)

7. Capgemini.: The User Challenge Benchmarking - The Supply of Online Public Services (2007)

8. Codagnone, C., Arne, U.T.: Benchmarking eGovernment: Tools, Theory, and Practice. European Journal of ePractice, 1-15 (August 2008)

9. Dattakumar, R., Jagadeesh, R.: A Review of Literature on Benchmarking. Benchmarking: An International Journal 10, 176-209 (2003)

10. Dorsch, J.J., Yasin, M.M.: A Framework for Benchmarking in the Public Sector Future Research. Benchmarking: An International Journal 11(2), 91-151 (1998)

11. Edwards, J.R.: Construct Validation in Organizational Behavior Research. In: Organizational Behavior: The State of the Science, pp. 327-371 (2003)

12. Engeström, Y.: The Future of Activity Theory: A Rough Draft. In: Sannino, A.L., Daniels, H., Gutierrez, K.D. (eds.) Learning and Expanding with Activity Theory. Cambridge University Press, Cambridge (2009)

13. Heeks, R.: iGovernment eGovernment: Improving the National and International Measurement, Evaluation and Comparison of eGovernment (2006)

14. Heeks, R.: eGovernment as a Carrier of Context. Journal of Public Policy 25, 51-74 (2005)

15. IANIS: Guide to Regional Good Practice Indicators and Benchmarking (2007)

16. Jansen, J., de Vries, S., van Schaik, P.: The Contextual Benchmark Method: Benchmarking e-Government Services. Government Information Quarterly 27(3), 213 219 (2010)

17. Janssen, M.: Measuring and Benchmarking the Back-end of E-Government: A Participative Self-assessment Approach. In: Wimmer, M.A., Chappelet, J.-L., Janssen, M., Scholl, H.J. (eds.) EGOV 2010. LNCS, vol. 6228, pp. 156-167. Springer, Heidelberg (2010)

18. Kouzmin, A., Löffler, E., Klages, H., Korac-Kakabadse, N.: Benchmarking and Performance Measurement in Public Sectors: Towards Learning for Agency Effectiveness. International Journal of Public Sector Management 12(2), 121-144 (1999)

19. Kyrö, P.: Revising the Concept and Forms of Benchmarking. Benchmarking: An International Journal 10(3), 210-225 (2003)

20. McGonagle, J.J., Fleming, D.: Option in Benchmarking. The Journal of Quality and Participation (March/April 1998)

21. Middendorp, C.P.: On the Conceptualization of Theoretical Constructs (1991) 
22. Muller, B., Ahlemann, F., Roeder, K.: Understanding the Success of Strategic IT / IS Benchmarking: Results from a Multiple-Case Study. In: 18th European Conference on Information Systems (2010)

23. Nardi, B.A.: Action Models, and Distributed Cognition. In: Context and Consciousness, Massachusetts Institute of Technology, Cambridge, MA, pp. 35-52 (1995)

24. Ojo, A., Janowski, T., Estevez, E.: Determining Progress towards e-Government: What Are the Core Indicators? In: 5th European Conference on e-Government, pp. 313-322. Academic Publishing International Ltd, New York (2005)

25. O'Leary, D.E.: Enterprise Ontologies: Review and an Activity Theory Approach. Internat. Journal of Accounting Information Systems 11(4), 336-352 (2010)

26. Paasi, M.: Benchmarking Policies. Science and Public Policy 32(1), 17-27 (2005)

27. Salem, F.: Benchmarking the e-Government Bulldozer: Beyond Measuring the Tread Marks. Journal of Measuring Business Excellence 11(4), 9-22 (2008)

28. Schellong, A.: EU e-Government Benchmarking 2010 EU e-Government Benchmarking General Remarks on the Future of Benchmarking Digital Government in the EU (2009)

29. Sharfman, M.P., Dean Jr, J.W.: Conceptualizing and Measuring the Organizational Environment: A Multidimensional Approach. Journal of Management 17(4), 681-700 (1991)

30. Vakkayil, J.D.: Activity Theory: A Useful Framework for Analysing Project-Based Organizations. Vikalpa 35(3), 1-18 (2010)

31. Yasin, M.M.: The Theory and Practice of Benchmarking: Then and Now. Benchmarking: An International Journal 9(3), 217-243 (2002) 